\title{
CircSKA3 Modulates FOXMI to Facilitate Cell Proliferation, Migration, and Invasion While Confine Apoptosis in Medulloblastoma via miR-383-5p
}

This article was published in the following Dove Press journal:

Cancer Management and Research

\section{Xinfang Wang* \\ Dong $\mathrm{Xu}^{*}$ \\ Xin Pei \\ Yingying Zhang \\ Yuling Zhang \\ Yaxing Gu \\ Ying Li}

Department of Pediatrics, Shandong Provincial Western Hospital, Jinan 250022, Shandong, People's Republic of China

*These authors contributed equally to this work
Correspondence: Ying Li Department of Pediatrics, Shandong Provincial Western Hospital, No. 4 Duanxing West Road, Jinan, Shandong 250022,

People's Republic of China

Tel +86-53I 83086400

Email dedfedx@163.com
Background: Medulloblastoma (MB) is the most common malignant brain tumor during childhood. Circular RNA (circSKA3) was identified to function as an oncogene in MB. However, the mechanism of circSKA3 in MB remains unclear.

Methods: The levels of circSKA3, microRNA-383-5p (miR-383-5p), and forkhead box M1 (FOXM1) in MB tissues were measured by quantitative real-time polymerase chain reaction (qRT-PCR). The cell viability and apoptotic rate were assessed via 3-(4,5-dimethyl-2-thiazolyl)-2,5-diphenyl-2-H-tetrazolium bromide (MTT) assay and flow cytometry, respectively. The protein levels of B-cell lymphoma 2 (Bcl-2), C-Caspase3, and FOXM1 were detected via Western blot assay. Cell cycle was detected by flow cytometry. The migration and invasion abilities were monitored by Transwell assay. The dual-luciferase reporter assay was constructed to verify the interactions between miR-383-5p and circSKA3 or FOXM1. The mice model experiment was carried out to validate the effects of circSKA3 in vivo.

Results: The levels of circSKA3 and FOXM1 were significantly elevated, while the level of miR-383-5p was notably declined in MB tissues. CircSKA3 was validated to sponge miR$383-5$ p and FOXM1 was a candidate target of miR-383-5p. CircSKA3 silencing impeded cell proliferation, migration, and invasion while promoted apoptosis by targeting miR-383-5p in vitro and retarded xenograft tumor growth in vivo. miR-383-5p suppressed cell proliferation, migration, and invasion but promoted apoptosis in MB cells by regulating FOXM1. CircSKA3 depletion decreased FOXM1 expression via miR-383-5p in MB cells.

Conclusion: CircSKA3 augmented MB progression partly through miR-383-5p/FOXM1 axis.

Keywords: circSKA3, miR-383-5p, FOXM1, medulloblastoma

\section{Introduction}

Medulloblastoma (MB) is a malignant brain tumor in children, ${ }^{1,2}$ with high heterogeneity and aggressive. MB consists of many different molecular subtypes. Based on the significant differences in genetics, demography, and clinical characteristics, MB was classified as four distinct molecular subtypes: WNT, SHH, Group 3, and Group 4. ${ }^{3,4}$ Current treatment methods including surgery, radiotherapy, and chemotherapy have contributed to the overall survival rate of $\mathrm{MB}$ patients, which the overall survival rates reached $75 \%{ }^{5,6}$ However, there is a high probability for $\mathrm{MB}$ survivors to suffer from long-term side effects, including neurological, 
neuroendocrine, and psychosocial deficits in the growing process of children. ${ }^{7,8}$ Therefore, it is urgent to search for novel therapeutic targets for MB patients.

With the development of next-generation sequencing methods, the aberrant expression of non-coding RNAs (ncRNAs) and their crucial roles in tumor were disclosed. ${ }^{9}$ Mounting research has suggested that ncRNAs are closely correlated with cancers including $\mathrm{MB}$, through regulating gene transcription, translation, and epigenetic modification, and then affected multiple biological processes. ${ }^{10-12}$ Circular RNAs (circRNAs) are a class of ncRNAs with a closed continuous loop without $5^{\prime}$ caps and $3^{\prime}$ tails. $^{13}$ The role of circRNAs has been extensively studied in various diseases. In recent years, the rapid evolution of high-throughput sequencing technique and bioinformatics helped us to realize that circRNAs was aberrantly expressed in mammalian cells and even higher than the linear RNA isoforms of its host genes. ${ }^{14}$ The dysregulated circRNAs were observed in various cancers including bladder carcinoma, colorectal cancer, ovarian cancer, as well as $\mathrm{MB}^{15-17}$ CircSKA3 (hsa_circ_0029696) was derived from the host gene Spindle And Kinetochore Associated Complex Subunit 3 (SKA3). A recent research exhibited that circSKA3 was upregulated in breast cancer tumors and cells, and its ectopic expression promoted tumor progression by complexing with Tks5 and integrin $\beta 1 .{ }^{18}$ Besides, research also showed that circSKA3 was highly expressed in MB, and circ-SKA3 could regulate cell proliferation, migration, and invasion in MB. ${ }^{17}$ This suggested that circ-SKA3 exerted pivotal oncogenic role in the evolution and progression of MB. However, the underlying mechanisms of circSKA3 in MB were unknown.

It is generally accepted that circRNAs functioned as "sponges" of miRNAs and participated in posttranscriptional regulation, and further reduced their ability to target mRNAs. ${ }^{19}$ MiRNAs are a form of small RNAs ( $\sim 22 \mathrm{nts})$ with no translation ability and mediate the messenger RNA (mRNA) expression or stability. ${ }^{20}$ Sufficient evidence showed that miRNAs were aberrantly expressed in MB. ${ }^{21,22}$ MiR-383-5p, a tumor suppressor, was reported to be downregulated in many human cancers including ovarian cancer, ${ }^{23}$ cervical cancer, ${ }^{24}$ and gastric carcinoma. ${ }^{25}$ However, no studies have yet disclosed its role in $\mathrm{MB}$.

Forkhead box M1 (FOXM1), a member of the Forkhead family of transcription factors, could bind to DNA sequence in the enhancer region of various target genes. ${ }^{26}$ FOXM1 was found to be involved in the development of many different types of tumor such as hepatocellular carcinoma ${ }^{27}$ and renal cell carcinoma. ${ }^{28}$ The pivotal role of FOXM1 in MB has been widely studied. FOXM1 was highly expressed in all subtypes of $\mathrm{MB}$, and its expression level was significantly correlated with the unfavorable clinical outcome of MB, which indicated that FOXM1 could be used as an additional prognostic marker and a potential novel target for MB treatment. ${ }^{29}$ Besides, knockdown of FOXM1 expression could inhibit cell proliferation and invasion, and induce apoptosis by caspase 3/ 7 pathway. ${ }^{30}$ However, the effect of FOXM1 in MB needs to be further illuminated.

In this research, we aimed to explore the underlying mechanism of circSKA3 in $\mathrm{MB}$, and this may provide a novel therapeutic target for MB patients.

\section{Materials and Methods}

\section{Tissue Samples Collection}

The study was permitted by the Ethics Committee of Shandong Provincial Western Hospital and executed according to the Declaration of Helsinki Principles (IRB No.2019SD662). Twenty tissue samples and corresponding adjacent normal tissue samples were obtained from Shandong Provincial Western Hospital. The clinicopathological features of subjects are exhibited in Table 1. All tissues were frozen at $-80^{\circ} \mathrm{C}$ until further used. Written informed consents were obtained from the adult patients, or from the parents or legal guardians of the child patients.

Table I The Clinicopathological Features of Medulloblastoma Patients

\begin{tabular}{|l|l|}
\hline Clinicopathological Features & Number of Cases \\
\hline Age(years) & 12 \\
$>3$ & 8 \\
$\leq 3$ & \\
\hline Gender & 14 \\
Male & 6 \\
Female & \\
\hline Histological subtype & 15 \\
Classic & 4 \\
Desmoplastic & 1 \\
Large cell/anaplastic & \\
\hline Metastasis at diagnosis & 18 \\
Absence & 2 \\
Presence & \\
\hline
\end{tabular}




\section{Quantitative Real-Time Polymerase Chain Reaction (qRT-PCR)}

The RNA in MB tissues or cells was extracted using TriQuick Reagent (Solarbio, Beijing, China). The reverse transcription was performed using a transcription kit (Solarbio), and the quantitative PCR was carried out using SYBR Premix Ex Taq II (TaKaRa, Dalian, China) on a 96-well Real-Time PCR Detection System (Bio-Rad, Shanghai, China). The levels of circSKA3, FOXM1, and miR-383-5p were normalized by glyceraldehyde 3-phosphate dehydrogenase (GAPDH) or small nuclear RNA U6, and then calculated by the $2^{-\Delta \Delta C t}$ method. The oligonucleotides of primers were obtained from Beijing Genomics Institute (BGI, Shenzhen, China) and exhibited as follows: circSKA3: (F， 5'-TGGGACTTCTGTACCATAAAGCAT -3', and R, 5'-ATCTATGGCCTCCTCACTGGT-3'); miR383-5p: (F, 5'-CGCGCGCAGATCAGAAGGTGA-3', and R, 5'-ATCCAGTGCAGGGTCCGAGG-3'); FOXM1: (F, 5'-ATGGCAAATTTTTCGCTCC-3', and R, 5'-ATG TCACCAGAAATTCCCAGTT-3'); GAPDH: (F, 5'-TGT TCGTCATGGGTGTGAAC-3', and R, 5'-ATG GCATGGACTGTGGTCAT-3'), and U6: (F, 5'-ATT GGAACGATACAGAGAAGATT-3', and R, 5'-GGA ACGCTTCACGAATTTG-3').

\section{Cell Culture and Transfection}

Two medulloblastoma cell lines DAOY and ONS-76 were purchased from Shanghai YaJi Biological (Shanghai, China). The cells were cultivated in Dulbecco's modified Eagle's medium (DMEM; Solarbio) containing 10\% fetal bovine serum (FBS; Solarbio) and 1\% penicillin/streptomycin (Solarbio) in a $5 \% \mathrm{CO}_{2}$ incubator at $37^{\circ} \mathrm{C}$.

Small interfering RNA (siRNA) against circSKA3 (sicircSKA3, final concentration, $20 \mathrm{nM}$ ) and matched negative control (si-NC, final concentration, $20 \mathrm{nM}$ ), miR-383-5p mimic (miR-383-5p , final concentration, $10 \mathrm{nM}$ ) and negative control (miR-NC, final concentration, $10 \mathrm{nM}$ ), miR-383$5 p$ inhibitor (anti-miR-383-5p, final concentration, $25 \mathrm{nM}$ ) and its scramble (anti-miR-NC, final concentration, $25 \mathrm{nM}$ ), siRNA targeting FOXM1 (si-FOXM1, final concentration, $20 \mathrm{nM}$ ) and its control (si-NC, final concentration, $20 \mathrm{nM}$ ) were synthesized in GenePharma (Shanghai, China). The fragments of FOXM1 were inserted into pcDNA3.1 (vector; Invitrogen, Carlsbad, CA, USA) to construct overexpression plasmid (FOXM1, final concentration, $1 \mu \mathrm{g} / \mathrm{mL}$ ). Cell transfection was carried out using Lipofectamine 2000 (Invitrogen) according to the manufacturer's instructions. In brief, $4 \times 10^{5}$ DAOY and ONS-76 cells were cultured for 24 $\mathrm{h}$ to grow to subconfluence, the miRNA mimic or inhibitor, siRNA, and plasmids were transfected into the cells. Twentyfour or 48 hours upon transfection, the cells were harvested for further research.

\section{RNase R Digestion}

Total RNA in MB cells was extracted using TriQuick Reagent (Solarbio, Beijing, China). Five micrograms of total RNA were incubated with RNase R (Epicenter Biotechnologies, Shanghai, China) at a concentration of $3 \mathrm{U} / \mu \mathrm{g}$. After total RNA incubation with RNase R for 15 min at $37^{\circ} \mathrm{C}$, the expression of circSKA3 was examined using qRT-PCR.

\section{Subcellular Localization}

Cytoplasmic \& Nuclear RNA Purification Kit (Norgen Biotek Corp., Belmont, MA, USA) was used to determine the localization of circSKA3. Cells were lysed by the Lysis Buffer J, and then cell lysates were centrifuged. Then, the nuclear RNA and cytoplasmic RNA were added into anhydrous ethanol and Buffer SK, respectively. Subsequently, the nuclear RNA and cytoplasmic RNA were eluted by the spin column. Finally, the proportion of circSKA3 in cytoplasmic and nucleus fractions was detected using qRTPCR.

\section{3-(4,5-Dimethyl-2-Thiazolyl)-}

\section{2,5-Diphenyl-2-H-Tetrazolium Bromide (MTT) Assay}

The cell viability of DAOY and ONS-76 cells was detected using MTT (Solarbio). The DAOY and ONS-76 cells $\left(3 \times 10^{3}\right.$ per well $)$ were firstly injected into a 96-well plate and cultivated for $24 \mathrm{~h}$. Following transfection, the cells were incubated for another $0 \mathrm{~h}, 24 \mathrm{~h}, 48 \mathrm{~h}$, and 72 h. MTT was injected into each well and maintained for 4 $\mathrm{h}$ at $37^{\circ} \mathrm{C}$. Then, dimethyl sulfoxide (DMSO) was added to dissolve the formazan for $10 \mathrm{~min}$ at $37^{\circ} \mathrm{C}$. Then, the absorbance was tested on a microplate reader.

\section{Flow Cytometry Analysis of Cell Apoptosis and Cell Cycle}

The apoptotic rate of DAOY and ONS-76 cells was assessed using Annexin V-fluorescein isothiocyanate (FITC)/propidium iodide (PI) apoptosis detection kit (Solarbio, Beijing, China). The DAOY and ONS-76 cells were firstly re-suspended in binding buffer and then 
incubated with Annexin V-FITC for $10 \mathrm{~min}$ in the dark. Then, the cell samples were incubated with PI for another $5 \mathrm{~min}$ in the dark. Within $1 \mathrm{~h}$, the apoptotic rate of cell samples was evaluated on flow cytometry. For detection of cell cycle, cells were stained with PI for $30 \mathrm{~min}$. Cell cycle distribution was detected by flow cytometry.

\section{Western Blot Assay}

Total protein in DAOY and ONS-76 cells was extracted using RIPA regent (Solarbio), and a protein assay kit (Beyotime, Shanghai, China) was utilized to measure the concentration of protein samples. Then, the protein samples (30 $\mu \mathrm{g} / \mathrm{lane})$ were separated by $12 \%-15 \%$ sodium dodecyl sulfonatepolyacrylamide gel electrophoresis (SDS-PAGE) and electrophoresed at $4^{\circ} \mathrm{C}$ for $1 \mathrm{~h}$ at a constant voltage of $70 \mathrm{~V}$ and another $1.5 \mathrm{~h}$ at a constant voltage of $110 \mathrm{~V}$. Then, the blots were transferred onto a polyvinylidene fluoride (PVDF) membrane (GE Healthcare, Piscataway, NJ, USA). Subsequently, the membrane was blocked with 5\% non-fat milk dissolved in TBST for $4 \mathrm{~h}$ at $37^{\circ} \mathrm{C}$ and incubated with primary antibodies: anti-B-cell lymphoma 2 (Bcl-2; ab32124, 1:1000; Abcam, Cambridge, MA, USA), C-Caspase3 (ab32042, 1:500; Abcam) or anti-GAPDH (ab8245, 1:10000; Abcam) overnight at $4^{\circ} \mathrm{C}$. Then, the membrane was incubated with goat antirabbit secondary antibody (ab216773, 1:10000; Abcam) for another $3 \mathrm{~h}$ at $37^{\circ} \mathrm{C}$. The primary antibodies were dissolved in $3 \%$ BSA with TBST, and the secondary antibody was dissolved in TBST. The intensity of bands was examined by an ECL kit (Beyotime).

\section{Transwell Assay}

The Transwell assay was conducted to detect the migrated and invaded abilities of DAOY and ONS-76 cells. For cell migration, the lower Transwell chamber (Corning, Tewksbury, MA, USA) was added with DMEM with $10 \% \mathrm{FBS}$, while the upper one was injected with DAOY and ONS-76 cells in DMEM without FBS. Following 24-h cultivation, the migrating cells on the backside of polycarbonate film were fixed with $4 \%$ methanol for $20 \mathrm{~min}$ and then stained with $0.1 \%$ violet crystal for $15 \mathrm{~min}$. The cells in 10 randomly selected fields were counted under a microscope. Original magnification, 200×.

For cell invasion, the protocols were similar to cell migration. While the difference is the upper chamber was coated with Matrigel matrix (BD Biosciences, San Jose, CA, USA).

\section{Dual-Luciferase Reporter Assay}

The wild type (containing the complementary binding sites) and mutant fragments of circSKA3 or 3'-untranslated regions
(3'UTR) of FOXM1 were cloned and inserted into pGL3 vector (Promega, Madison, WI, USA) to construct the dualluciferase reporter, named as circSKA3 WT, circSKA3 MUT, FOXM1 3'UTR WT, or FOXM1 3'UTR MUT. The cotransfection of luciferase reporter and miR-NC or miR-383$5 p$ was performed using Lipofectamine 2000 (Invitrogen). The luciferase activity was evaluated using Dual-Lucy Assay Kit (Solarbio).

\section{Mice Xenograft Models}

The nude mice experiment was performed according to the procedures approved by the Animal Care Committee of Shandong Provincial Western Hospital. Animal studies were performed in compliance with the ARRIVE guidelines and the Basel Declaration. All animals received humane care according to the National Institutes of Health (USA) guidelines. The DAOY cells $\left(5 \times 10^{6}\right)$ stably transfected with sh-NC or shcircSKA3 were injected subcutaneously into the right flank of the six-week-old male nude mice ( $n=6$ per group). Following injection, the xenograft tumor was measured every 7 days for 5 times and calculated according to the formula: volume $\left(\mathrm{mm}^{3}\right)=$ width $^{2} \times$ length $/ 2$. At 35 -d measurement, the xenograft tumors were resected from nude mice. The xenograft tumors' weight was measured, and the tumors were stored for the next exploration.

\section{Statistical Analysis}

GraphPad Prism 7 (GraphPad Inc., La Jolla, CA, USA) was used to perform the experiment data. All quantitative data were presented as the mean \pm standard deviation (SD) of at least three biological replicates carried out at the same time. Sample size ("n") always represents biological replicates. The differences between the two groups were processed by Student's $t$-test, and the differences among multiple groups were evaluated by one-way analysis of variance (ANOVA). Statistical significance was defined as $P$ value $<0.05$.

\section{Results}

\section{CircSKA3 Was Significantly Increased in MB Tissues}

In order to explore the role of circSKA3 in $\mathrm{MB}$, the level of circSKA3was firstly measured in MB tissues. As presented in Figure 1A, the level of circSKA3 was dramatically upregulated in $\mathrm{MB}$ tissues compared to that in adjacent normal tissues. Moreover, the results confirmed that circSKA3 was indeed circRNA, which was resistant to RNase $\mathrm{R}$ digestion (Figure 1B and C). Research has shown that circRNAs located 
in the nucleus mainly regulate the transcription of the parental gene, while in the cytoplasm, circRNAs could be used as endogenous competitive RNAs (ceRNA), thereby regulating the physiological activities of tumor cells. ${ }^{31}$ Subsequently, we measured the subcellular localization of circSKA3 by nuclear and cytoplasmic separation experiments. The result suggested that circSKA3 was mostly located in the cytoplasm of DAOY and ONS-76 cells (Figure 1D and E). These data indicated that circSKA3 was a circRNA and was upregulated in MB.

\section{CircSKA3 Knockdown Suppressed Cell Proliferation, Migration, and Invasion but Induced Apoptosis in DAOY and ONS-76 Cells}

To explore the functions of circSKA3 in MB, si-circSKA3 was transfected into DAOY and ONS-76 cells. As exhibited in Figure 2A and B, transfection of si-circSKA3 induced a near $50 \%$ reduction in circSKA3 expression in DAOY and ONS-76 cells in contrast with the si-NC group. The following MTT assay indicated that the transfection of si-circSKA3 in DAOY and ONS-76 cells resulted in a near 30\% reduction of cell viability related to that in the si-NC group (Figure 2C and D). However, the apoptosis rate was increased by $15 \%$ in DAOY and ONS-76 cells transfected with si-circSKA3
(Figure 2E and F). Since Bcl-2 and C-Caspase3 as apoptosisrelated markers, we detected levels of these proteins in sicircSKA3-transfected DAOY and ONS-76 cells. As showed in Figure $2 \mathrm{G}$ and $\mathrm{H}$, the protein level of $\mathrm{Bcl}-2$ was decreased by half, while the protein level of C-Caspase 3 was 3-4 fold enhanced in DAOY and ONS-76 cells transfected with sicircSKA3. Moreover, the proportion of the S-phase cells was reduced, while the G1/G0-phase cells were increased after circSKA3 knockdown (Figure 2I and J). Besides, the number of migration and invasion cells was both reduced in DAOY and ONS-76 cells transfected with si-circSKA3 (Figure 2K and $\mathrm{L}$ ). These results demonstrated that the depletion of circSKA3 blocked MB progression in vitro.

\section{MiR-383-5p Was Negatively Interacted with circSKA3 in DAOY and ONS-76 Cells}

As shown in Figure 3A and B, circSKA3 was enriched by circSKA3 probe in both DAOY and ONS-76 cells. To investigate the biological mechanism of circSKA3 in MB, starBase was used to search the putative targets of circSKA3. Venn diagram showed that miR-382-5p, miR383-5p, miR-520g-3p, miR-520h, and miR-576-5p harbored the binding sites of circSKA3 (Figure 3C). Moreover, miR-
A

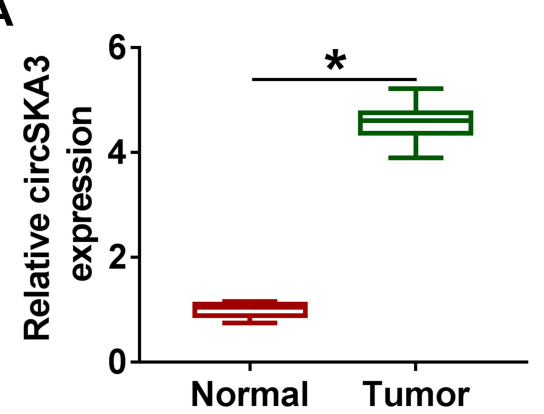

D

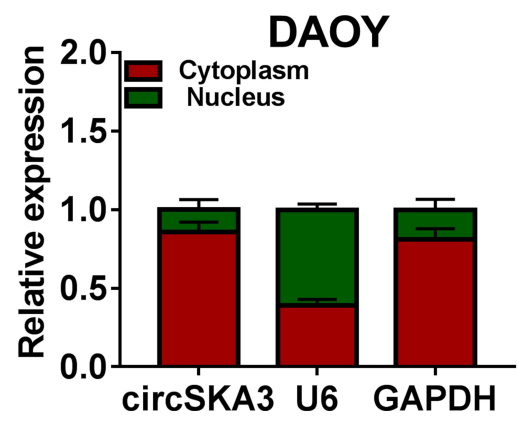

B

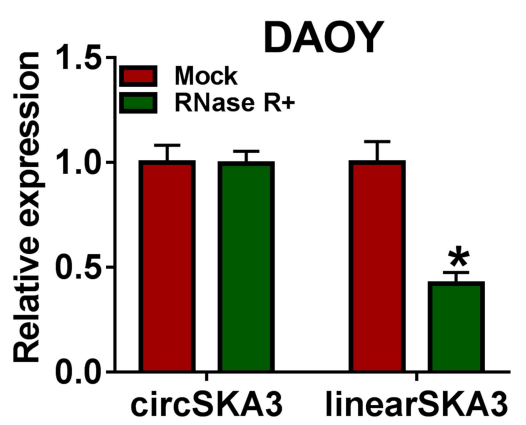

E

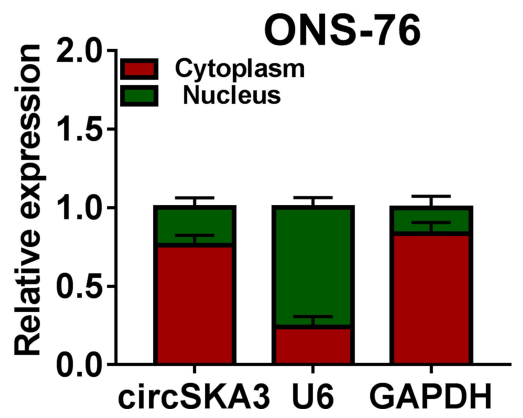

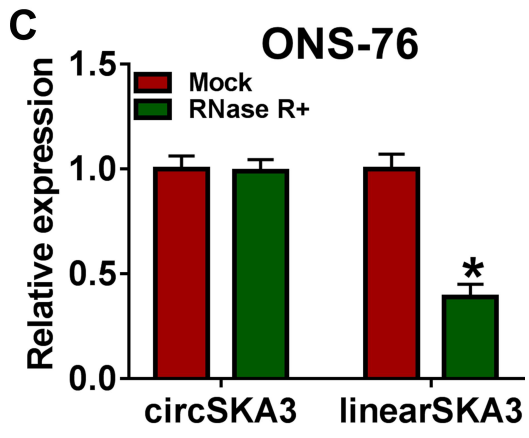

circSKA3 linearSKA3

Figure I CircSKA3 was significantly increased in MB tissues. (A) The level of circSKA3 in 20 MB tissues and adjacent normal tissues was tested by qRT-PCR. (B and C) CircSKA3 resistance to RNase R was detected by qRT-PCR. (D and E) QRT-PCR was used to assess the level of cytoplasmic control transcript (GAPDH), nuclear control transcript (U6) and circSKA3 in nuclear and cytoplasmic fractions. Error bar, SD; $n=3$ biological replicates. $* P<0.05$. 


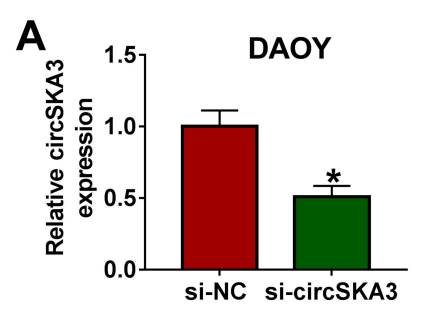

E

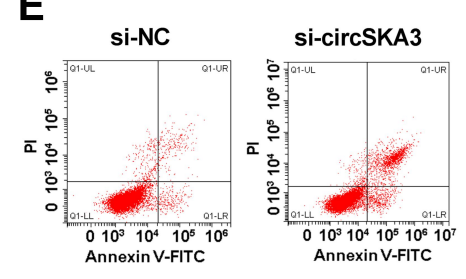

G
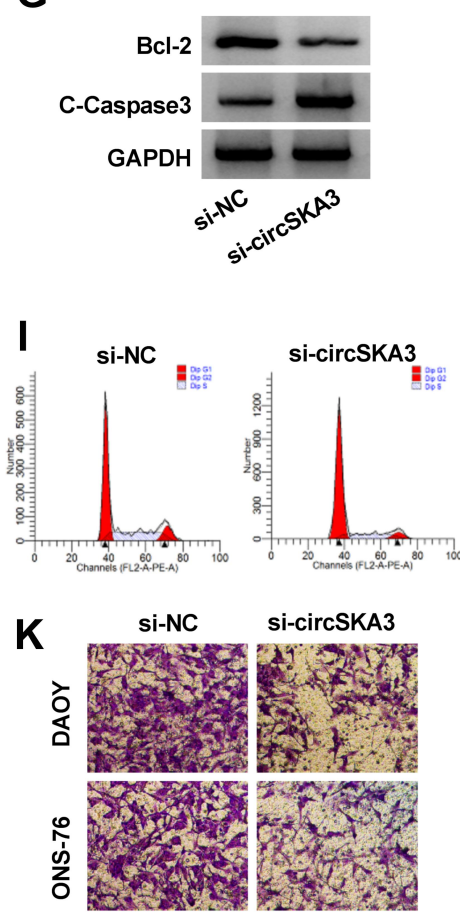
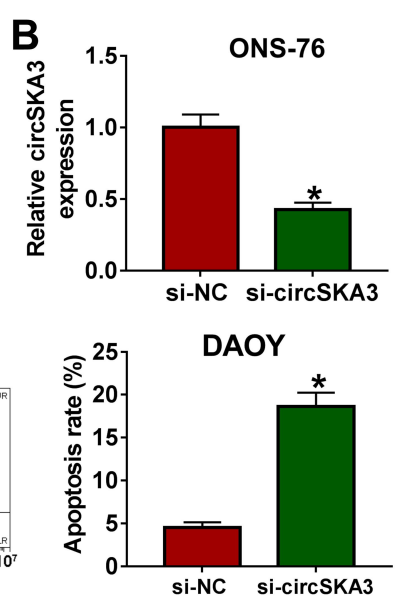

DAOY
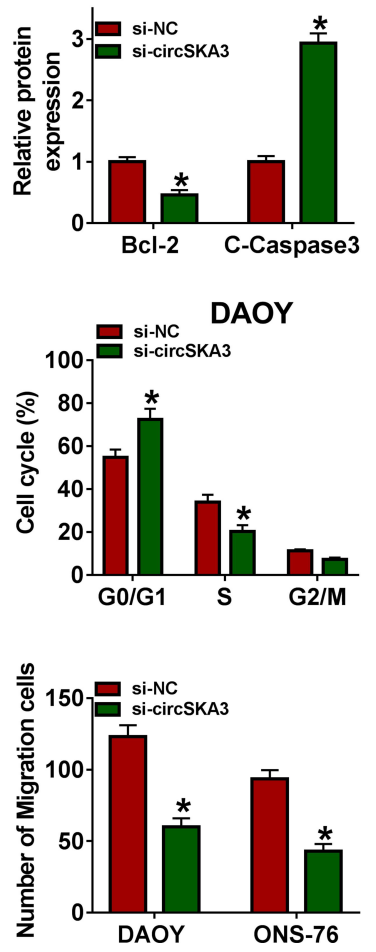

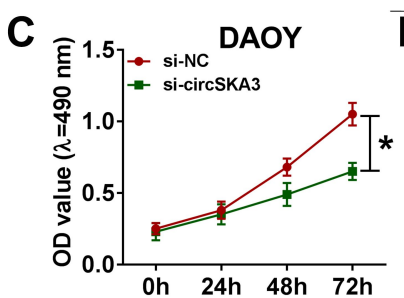

F
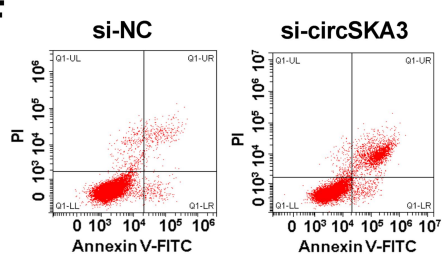

H
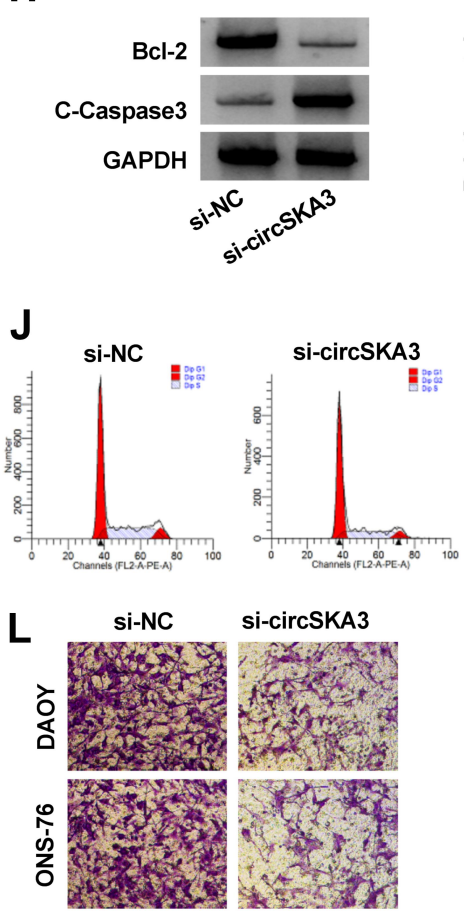
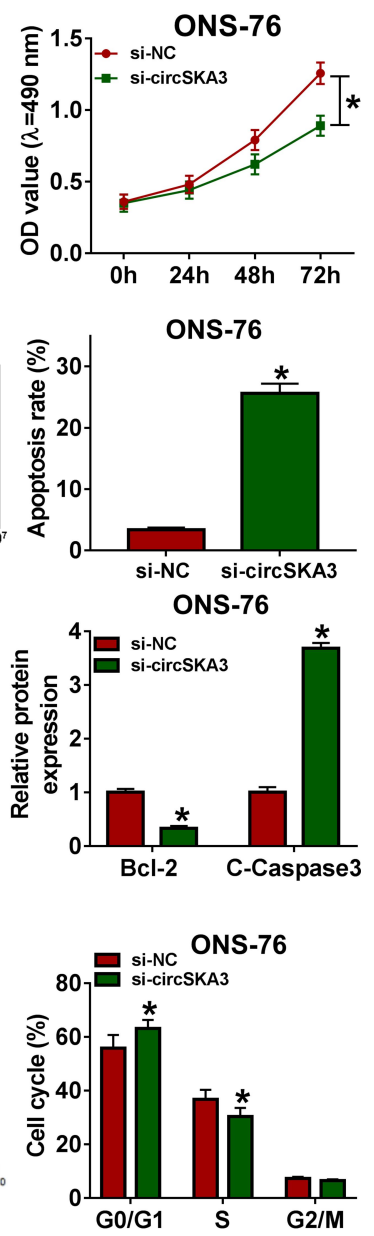

ONS-76

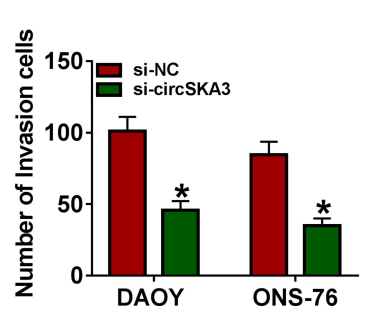

Figure 2 CircSKA3 knockdown suppressed cell proliferation, migration, and invasion but induced cell apoptosis and cycle arrest in DAOY and ONS-76 cells. (A-L) The DAOY and ONS-76 cells were transfected with si-NC or si-circSKA3. (A and B) The level of circSKA3 was tested by qRT-PCR. (C and D) The cell viability was examined via MTT assay. (E and F) The apoptotic rate was measured through flow cytometry. ( $\mathbf{G}$ and $\mathbf{H}$ ) The protein levels of Bcl-2 and C-Caspase3 were assessed by Western blot assay. ( $\mathbf{I}$ and $\mathbf{J})$ Cell cycle distribution was determined by flow cytometry. ( $\mathbf{K}$ and $\mathbf{L}$ ) The migration and invasion abilities were detected by Transwell assay. Error bar, SD; $\mathrm{n}=3$ biological replicates. $* P<0.05$.

382-5p, miR-383-5p, miR-520g-3p, miR-520h, and miR576-5p were captured by circSKA3 probe, and then the enrichment of miRNAs was detected by qRT-PCR. As shown in Figure 3D and E, miR-383-5p exhibited the highest expression, which indicated that circSKA3 exhibited the strongest interaction with miR-383-5p. Thus, miR-383-5p was selected for further research. As presented in Figure 3F, miR-383-5p had complementary biding sites with circSKA3. Following dual-luciferase reporter assay implied that the transfection of miR-383-5p contributed to the notable decline of luciferase activity of circSKA3 WT reporter in contrast to that in the miR-NC group, while the luciferase activity of circSKA3 MUT reporter had no change in any group (Figure 3G and H). In addition, the level of miR-383$5 p$ was obviously increased in si-circSKA3-transfected DAOY and ONS-76 cells (Figure 3I). Besides, the data indicated that miR-383-5p was significantly decreased in MB tissues (Figure 3J). These data disclosed that miR-383$5 p$ was decreased in DAOY and ONS-76 cells and was a target of circSKA3 in MB tissues. 

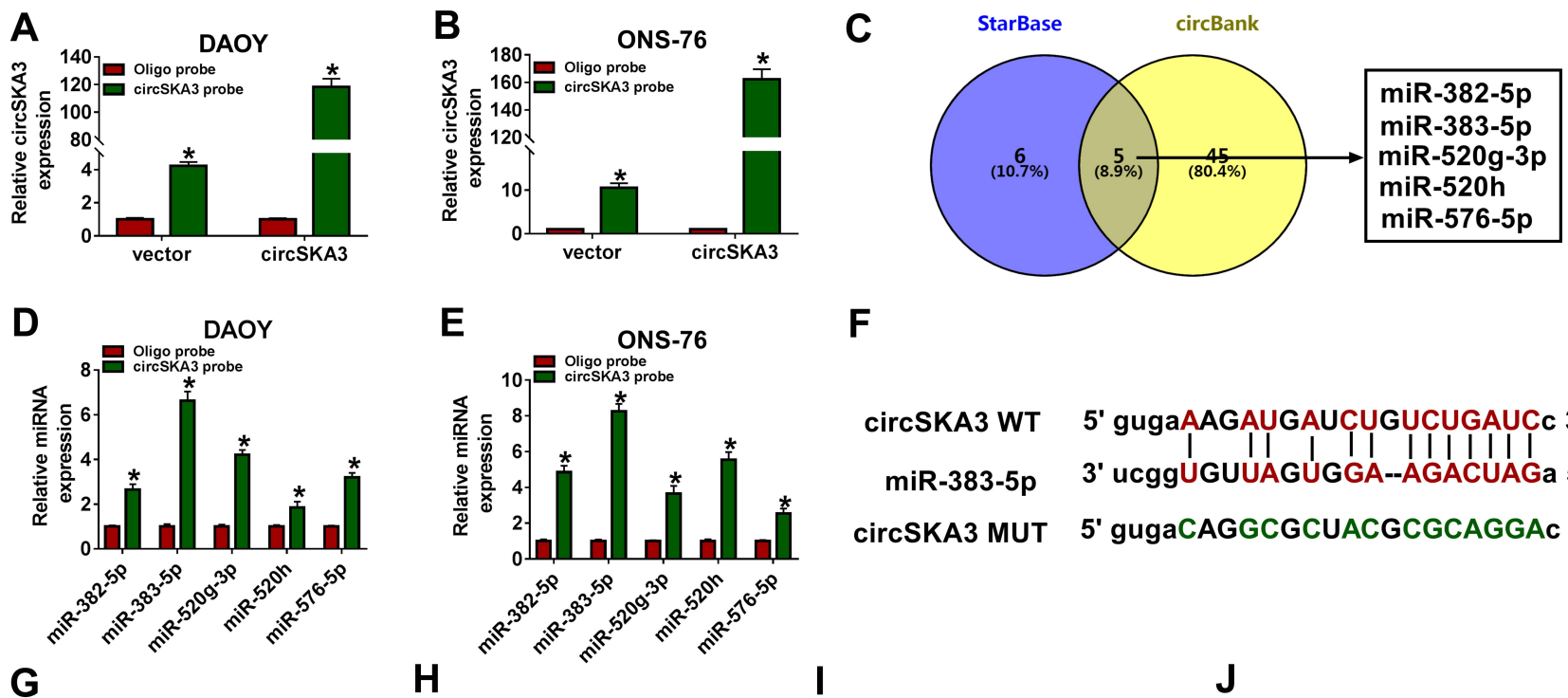

$\mathbf{F}$
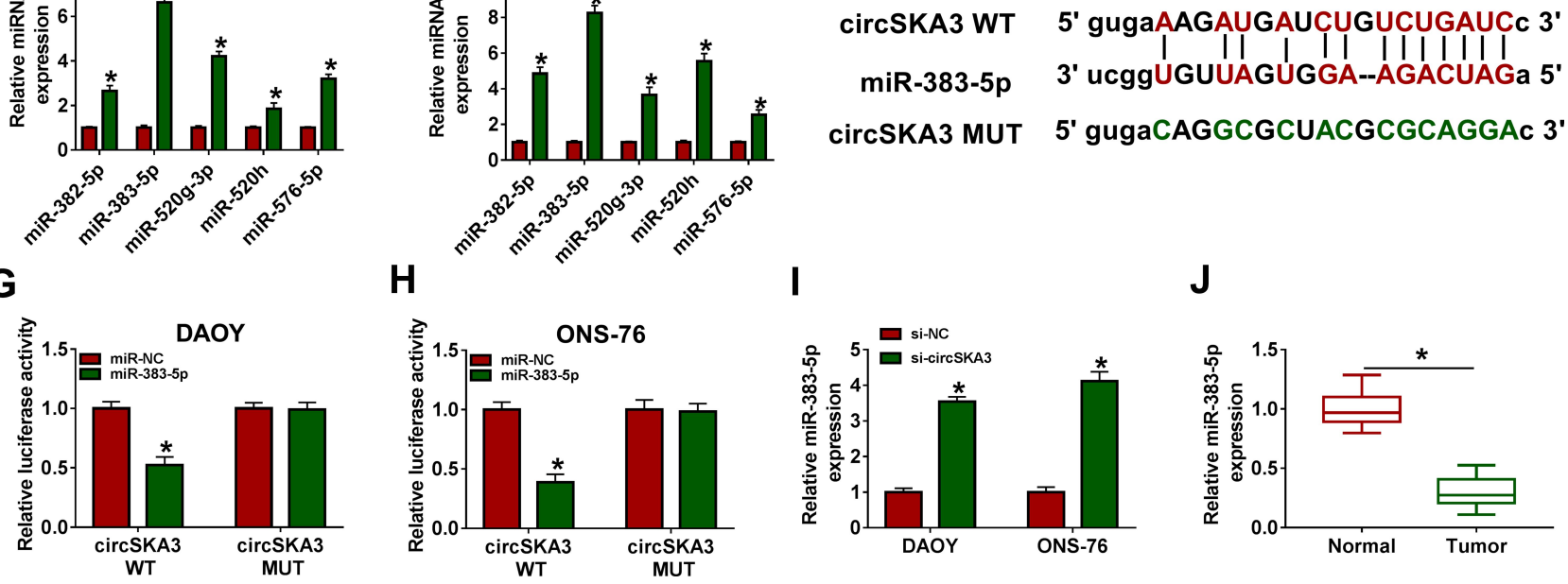

I

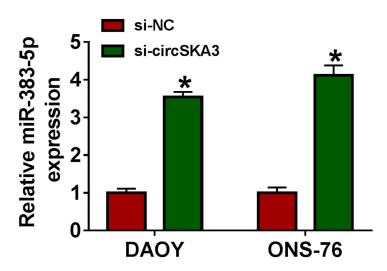

$\mathbf{J}$

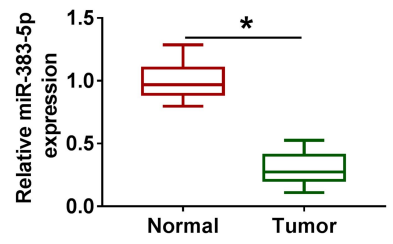

Figure 3 miR-383-5p was negatively interacted with circSKA3 in DAOY and ONS-76 cells. (A and B) The expression of circSKA3 was detected by qRT-PCR. (C) Venn diagram showed that miR-382-5p, miR-383-5p, miR-520g-3p, miR-520h and miR-576-5p contained the binding sites of circSKA3. (D and E) The enrichment of miR-382-5p, miR-383-5p, miR-520g-3p, miR-520h and miR-576-5p was detected RNA pull down assay using circSKA3 probe. (F) The complementary binding sites between miR-383-5p and circSKA3 were displayed, as well as the mutant sequences of circSKA3. (G and $\mathbf{H}$ ) The luciferase activity of circSKA3 WT or circSKA3 MUT reporter in DAOY and ONS-76 cells transfected with miR-NC or miR-383-5p was detected via dual-luciferase reporter assay. (I) The level of miR-383-5p in DAOY and ONS-76 cells transfected with si-NC or si-circSKA3 was tested by qRT-PCR. (J) The level of miR-383-5p in 20 MB tissues and adjacent normal tissues was tested by $q R T-P C R$. Error bar, SD; $n=3$ biological replicates. ${ }^{*} P<0.05$.

\section{CircSKA3 Facilitated Cell Proliferation, Migration, and Invasion While Repressed Apoptosis in DAOY and ONS-76 Cells by Regulating miR-383-5p}

To explore the functions of circSKA3 and miR-383-5p in MB, anti-miR-383-5p and si-circSKA3 were co-transfected into DAOY and ONS-76 cells. As exhibited in Figure 4A and B, the level of miR-383-5p was evidently up-regulated in DAOY and ONS-76 cells transfected with si-circSKA3, while the introduction of miR-383-5p inhibitor mitigated the promotion effect on miR-383-5p. The absence of miR-383-5p relieved the cell viability in DAOY and ONS-76 cells inhibited by circSKA3 knockdown (Figure 4C and D). While the apoptotic rate was notably elevated in DAOY and ONS-76 cells transfected with si-circSKA3, but the transfection of miR-383-5p inhibitor attenuated this promotion effect (Figure 4E and F). Furthermore, miR-383-5p inhibitor weakened the inhibitory impact on the protein level of Bcl-2, as well as the accelerated impact on the protein level of C-Caspase 3 in DAOY and ONS-
76 cells caused by circSKA3 deletion (Figure 4G and H). Moreover, circSKA3 knockdown-induced promotion effect on G1/G0-phase cells and inhibition effect on S-phase cells were partially reversed by miR-383-5p inhibitor (Figure 4I and J). Besides, the migrated and invaded abilities were apparently declined in DAOY and ONS-76 cells transfected with sicircSKA3, while partly regained by the re-introduction of antimiR-383-5p (Figure $4 \mathrm{~K}$ and L). These data uncovered that circSKA3 accelerated MB progression in DAOY and ONS-76 cells by targeting miR-383-5p.

\section{FOXM I Was a Direct Target of miR-383- $5 p$ in DAOY and ONS-76 Cells}

To illustrate the mechanism of miR-383-5p in MB, TargetScan was utilized to predict the potential targets of miR-383-5p. As presented in Figure 5A, the 3'UTR of FOXM1 had complementary sequences with miR383-5p. The luciferase activity of FOXM1 3'UTR WT reporter was significantly decreased in DAOY and ONS- 


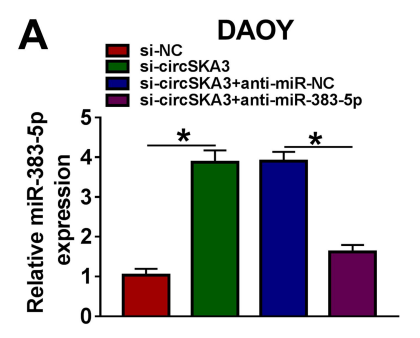

E
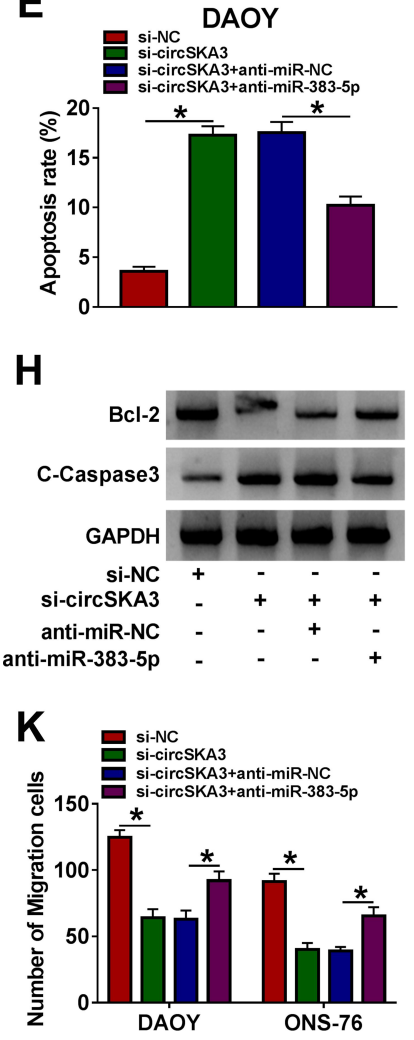

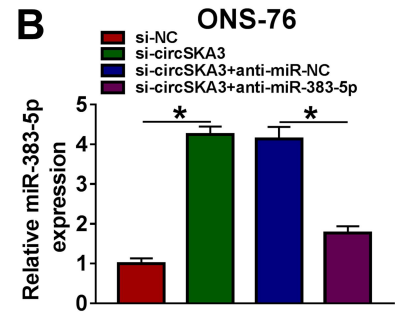

$\mathbf{F}$
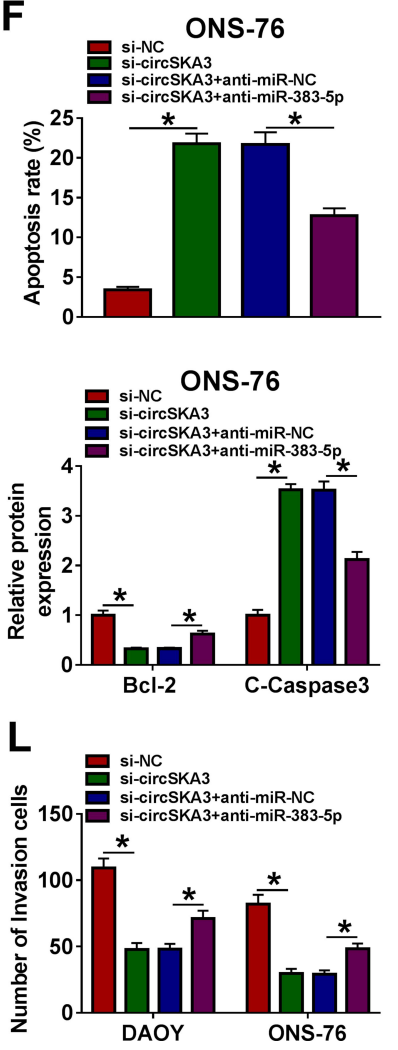

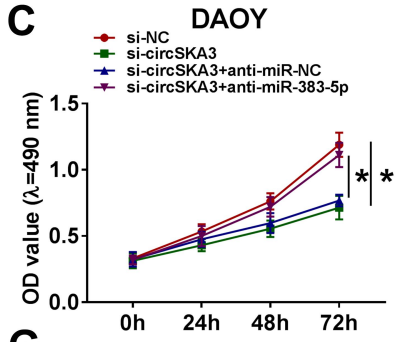

G

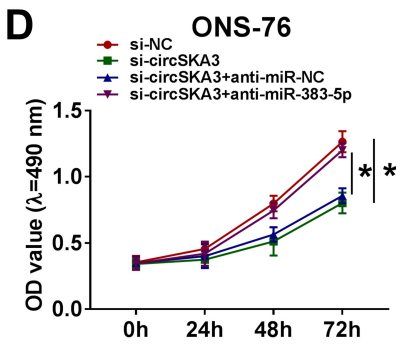

DAOY
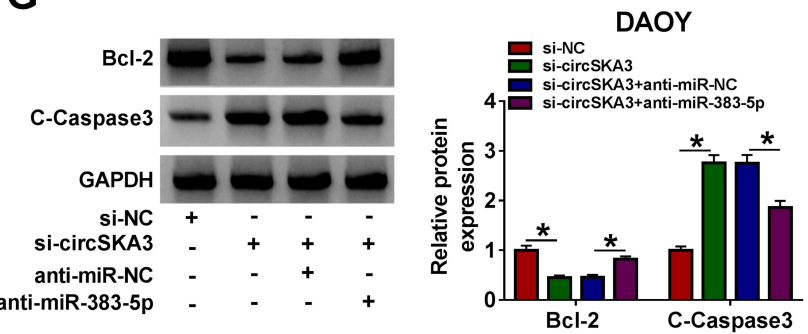

I
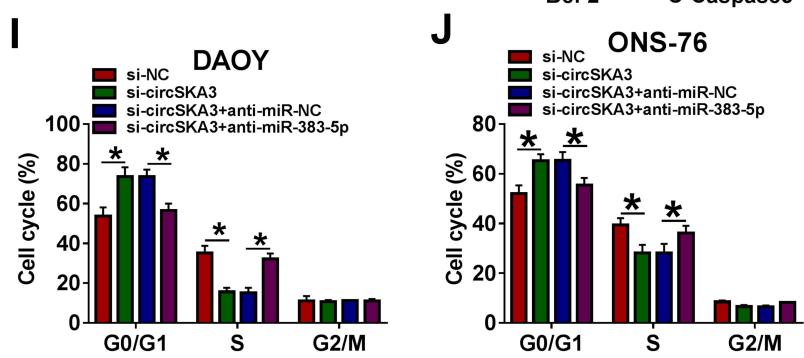

Figure 4 CircSKA3 facilitated cell proliferation, migration, and invasion while repressed apoptosis in DAOY and ONS-76 cells by regulating miR-383-5p. (A-L) The DAOY and ONS-76 cells were transfected with si-NC, si-circSKA3, si-circSKA3 + anti-miR-NC, si-circSKA3 + anti-miR-383-5p. (A and B) The level of miR-383-5p was examined by qRT-PCR. ( $\mathbf{C}$ and $\mathbf{D})$ The cell viability was monitored via MTT assay. ( $\mathbf{E}$ and $\mathbf{F}$ ) The apoptotic rate was assessed through flow cytometry. ( $\mathbf{G}$ and $\mathbf{H})$ The protein levels of Bcl-2 and C-Caspase3 were evaluated by Western blot assay. (I and $\mathbf{J}$ ) Cell cycle arrest was measured by flow cytometry. (K and $\mathbf{L})$ The migration and invasion abilities were tested by Transwell assay. Error bar, SD; $\mathrm{n}=3$ biological replicates. ${ }^{*} \mathrm{P}<0.05$.

76 cells transfected with miR-383-5p, while the luciferase activity FOXM1 3'UTR MUT reporter had no apparent fluctuation in any group (Figure 5B and $\mathrm{C}$ ). Moreover, the protein expression of FOXM1 was suppressed by miR-383-5p in DAOY and ONS-76 cells (Figure 5D). The protein level of FOXM1 was remarkably reduced in DAOY and ONS-76 cells transfected with si-circSKA3, but partially regained in DAOY and ONS-76 cells co-transfected with si-circSKA3 and antimiR-383-5p (Figure 5E). Besides, the mRNA and protein levels of FOXM1 were both conspicuously enhanced in $\mathrm{MB}$ tissues (Figure $5 \mathrm{~F}$ and $\mathrm{G}$ ). Taken together, FOXM1 negatively interacted with miR-383$5 \mathrm{p}$ in DAOY and ONS-76 cells.
MiR-383-5p Confined Cell Proliferation, Migration, and Invasion While Facilitated Apoptosis in DAOY and ONS-76 Cells by Targeting FOXMI

To further explore the functions of miR-383-5p and FOXM1 in MB, miR-383-5p and FOXM1 were co-transfected into DAOY and ONS-76 cells. As displayed in Figure 6A and B, the protein level of FOXM1 was notably declined in miR383-5p-transfected DAOY and ONS-76 cells, while recovered in DAOY and ONS-76 cells co-transfected with miR383-5p and FOXM1. The MTT assay and Transwell assay results indicated that the transfection of FOXM1 overexpression rescued the cell viability, migrated, and invaded abilities 

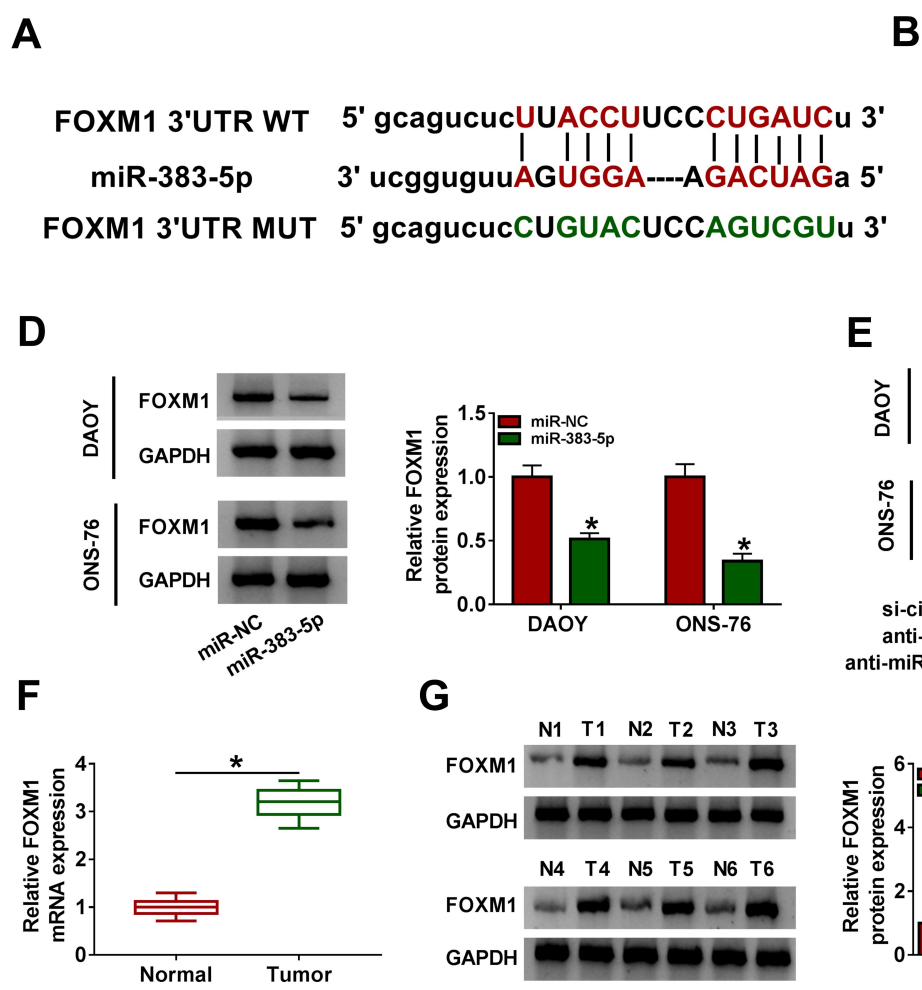

$\mathbf{E}$

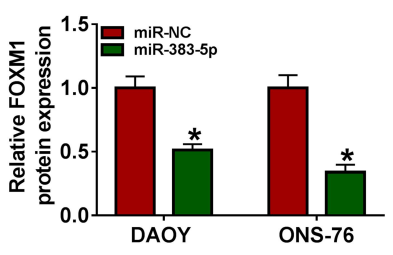

G

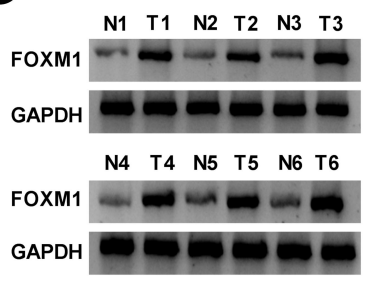

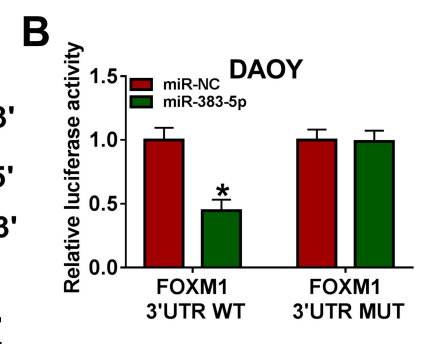
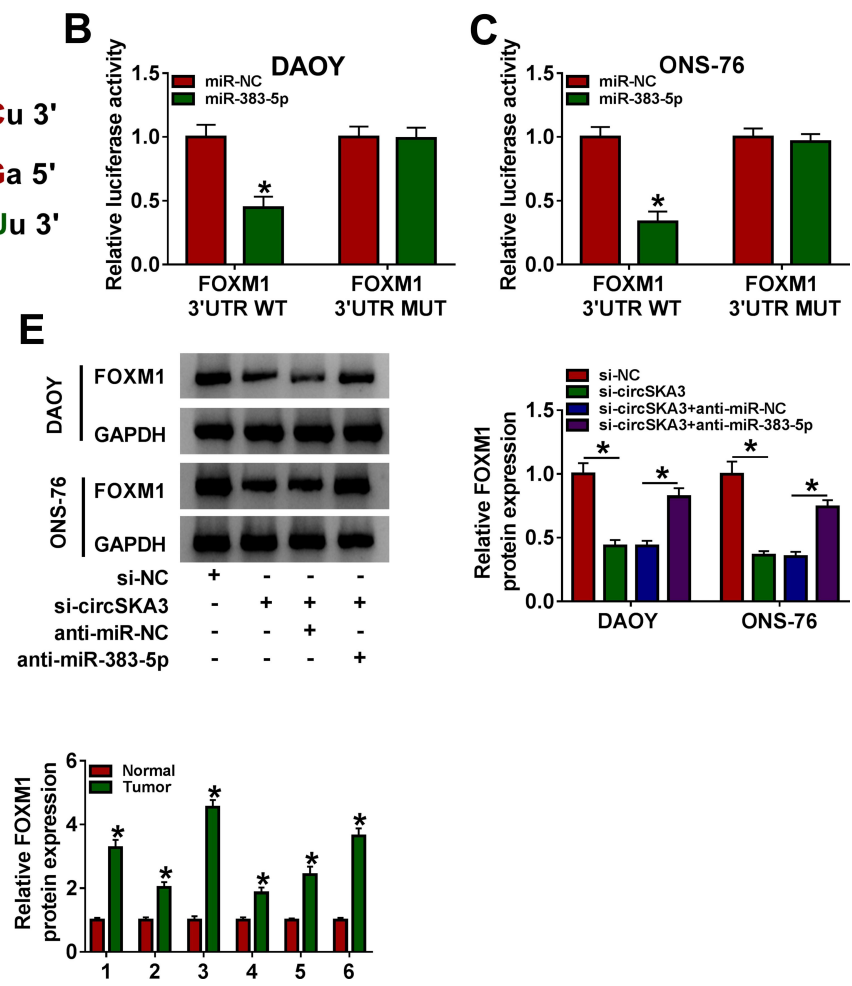

Figure 5 FOXMI was a direct target of miR-383-5p in DAOY and ONS-76 cells. (A) The complementary binding sites between miR-383-5p and FOXMI were exhibited, as well as the mutant sequences of FOXMI. (B and C) The luciferase activity of FOXMI 3'UTR WT or FOXMI 3'UTR MUT reporter in DAOY and ONS-76 cells transfected with miR-NC or miR-383-5p was detected via dual-luciferase reporter assay. (D) The protein level of FOXMI in DAOY and ONS-76 cells transfected with miR-NC or miR383-5p was tested by Western blot assay. (E) The protein level of FOXMI in DAOY and ONS-76 cells transfected with si-NC, si-circSKA3, si-circSKA3 + anti-miR-NC or sicircSKA3 + anti-miR-383-5p was determined by Western blot. (F) The mRNA level of FOXMI was detected via qRT-PCR. (G) The protein level of FOXMI was assessed by Western blot assay. Error bar, SD; $n=3$ biological replicates. $* P<0.05$.

in DAOY and ONS-76 cells constrained by miR-383-5p (Figure 6C and D and $\mathrm{K}$ and $\mathrm{L}$ ). However, the apoptotic rate and cell cycle showed the opposite trends. Briefly, the apoptotic rate was distinctly increased in the miR-383-5p group, while partially receded in miR-383-5p + FOXM1 group (Figure 6E and F). Furthermore, the introduction of FOXM1 upregulation counteracted the restraint effect on the protein level of Bcl-2 and the promoted effect on the protein level of C-Caspase 3 in DAOY and ONS-76 cells caused by miR-383-5p overexpression (Figure 6G and H). Besides, miR-383-5p overexpression-induced cell cycle arrest was abolished by FOXM1 overexpression (Figure 6I and J). To sum up, miR-383-5p suppressed MB progression by regulating FOXM1.

\section{CircSKA3 Silencing Confined Xenograft Tumor Growth in vivo}

To further validate the effects of circSKA3 in MB, the mice model experiment was carried out. As presented in Figure $7 \mathrm{~A}$ and $\mathrm{B}$, the volume and weight of xenograft tumor were both markedly reduced in the sh-circSKA3 group in comparison with that in the sh-NC group. The level of circSKA3 was obviously down-regulated, but miR-383-5p was notably enhanced in the sh-circSKA3 group (Figure 7C and D). In addition, the protein level of FOXM1 was strikingly decreased in the sh-circSKA3 group (Figure 7E). These data unraveled that circSKA3 knockdown blocked xenograft tumor growth in vivo.

\section{Discussion}

Tumor progression is a complicated process in the human body. CircSKA3 was reported to implicate in tumor progression in MB. This research aimed to investigate the mechanism and functions of circSKA3 in MB. Our research demonstrated that circSKA3 knockdown restrained MB progression partially through miR-383-5p/ FOXM1 axis.

The ectopic expression of circSKA3 was associated with tumor progression. For example, Du et al reported that circSKA3 was dramatically increased in breast cancer, and its silencing constrained the invasive capacity of breast cancer cells in vitro and in vivo by binding integrin 
A

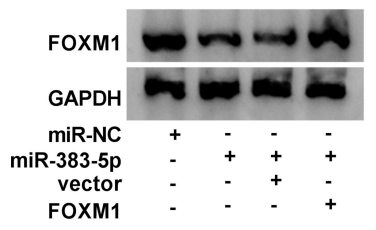

C

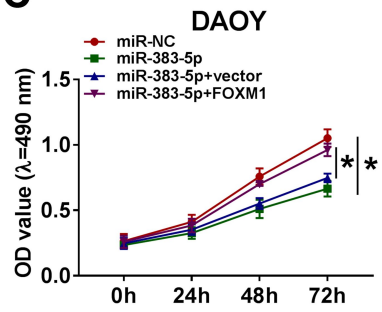

G

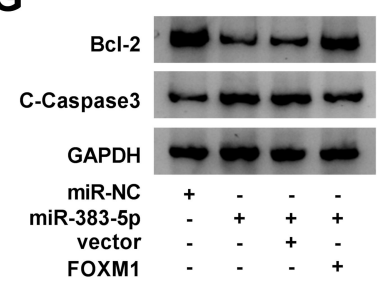

I

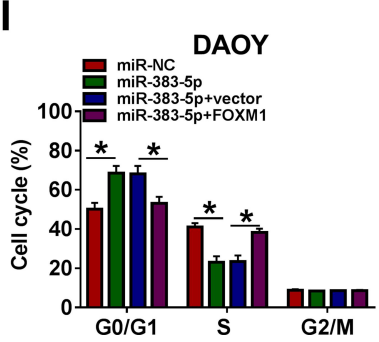

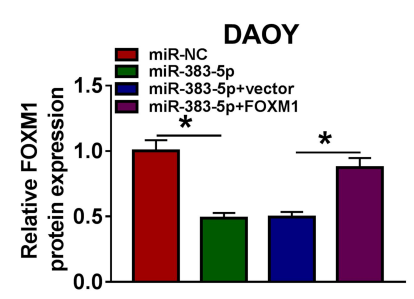

D
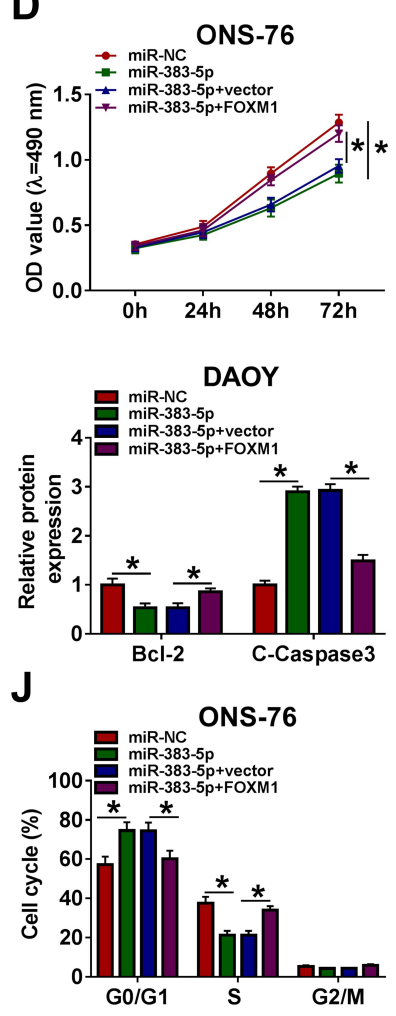

B

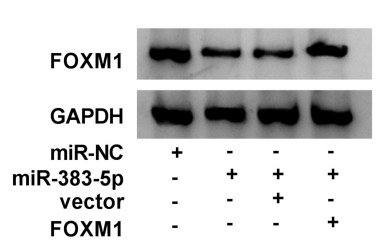

E

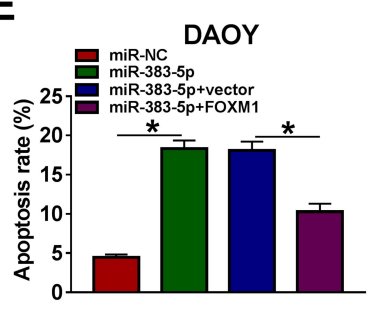

H

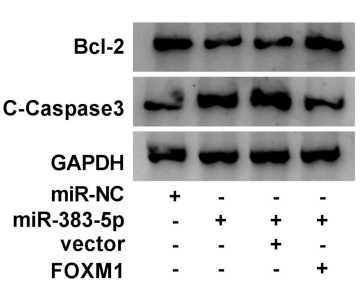

K

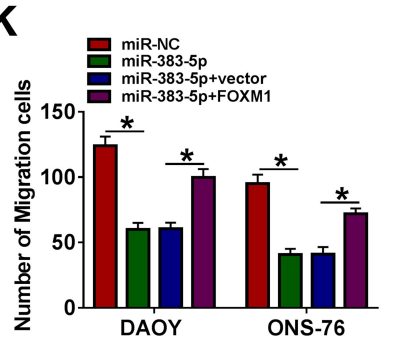

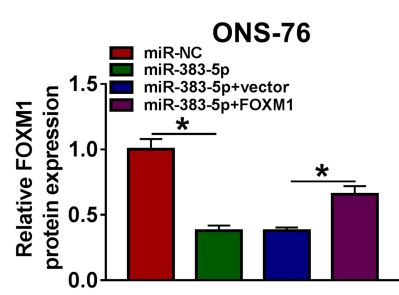

$\mathbf{F}$
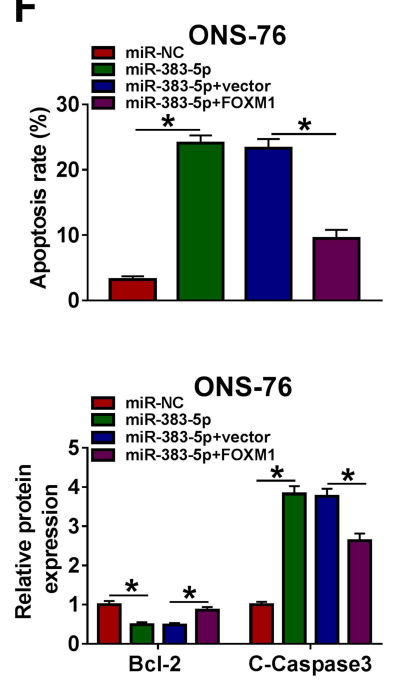

$\mathbf{L}$

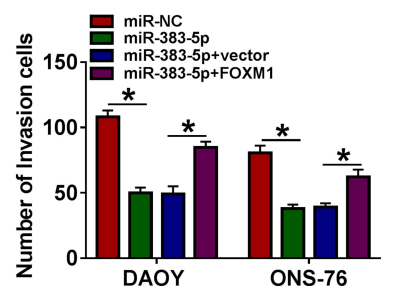

Figure 6 miR-383-5p confined cell proliferation, migration, and invasion while facilitated apoptosis in DAOY and ONS-76 cells by targeting FOXMI. (A-J) The DAOY and ONS-76 cells were transfected with miR-NC, miR-383-5p, miR-383-5p + vector, or miR-383-5p + FOXMI. (A and B) The protein level of FOXMI was examined by Western blot. (C and $\mathbf{D})$ The cell viability was monitored via MTT assay. (E and $\mathbf{F}$ ) The apoptosis rate was measured through flow cytometry. (G and $\mathbf{H})$ The protein levels of Bcl-2 and C-Caspase3 were detected by Western blot assay. (I and $\mathbf{J}$ ) Cell cycle progression was determined by flow cytometry. (K and $\mathbf{L}$ ) The migration and invasion abilities were evaluated by Transwell assay. Error bar, $S D ; n=3$ biological replicates. $* P<0.05$.

$\beta 1 .{ }^{18} \mathrm{~A}$ recent study indicated that circSKA3 was involved in the progression of $\mathrm{MB} .{ }^{17}$ In the current study, we found that circSKA3 was notably enhanced in MB. Furthermore, the silencing of circSKA3 curbed cell proliferation, metastasis but induced apoptosis. Besides, circSKA3 depletion restrained xenograft tumor growth in vivo. These data revealed that circSKA3 accelerated $\mathrm{MB}$ progression.

Convincing evidence elucidated that miR-383-5p was involved in tumor progression in many types of cancers. For instance, a study in ovarian cancer indicated that miR383-5p was reduced in ovarian cancer, and its overexpression regulated cell behaviors and chemo-sensitivity by targeting TRIM27. ${ }^{32}$ Another study demonstrated that miR-383-5p was down-regulated in gastric cancer, and overexpression of miR-383-5p impeded cell growth and metastasis. $^{33}$ In our research, miR-383-5p was downregulated in $\mathrm{MB}$ tissues, and it was predicted as a target of circSKA3. The expression of miR-383-5p was suppressed by circSKA3 depletion. Besides, miR-383-5p inhibitor partly reversed the inhibition effects on cell viability, cell cycle progression, migration and invasion, as well as the promotion effect on cell apoptosis induced by sicircSKA3. These results unraveled that circSKA3 facilitated MB progression by suppressing miR-383-5p.

FOXM1 was related to tumor progression in diverse cancers. Wang et al implied that FOXM1 was enhanced in human non-small cell lung cancer, and its overexpression boosted cell growth and metastasis in vitro. ${ }^{34}$ Another 

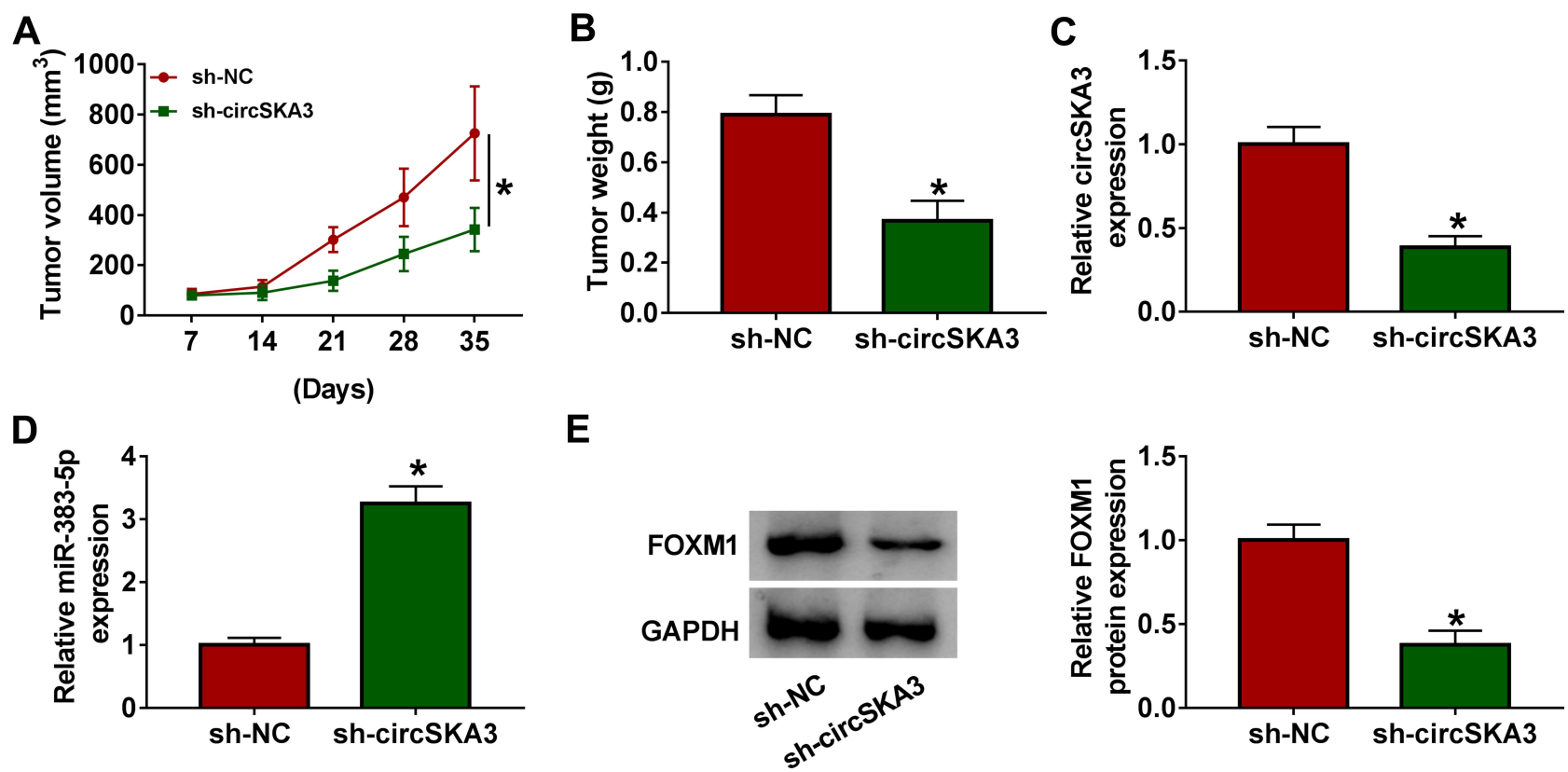

Figure 7 CircSKA3 silencing confined xenograft tumor growth in vivo. (A-E) The six-week-old male nude mice ( $\mathrm{n}=6$ per group) were injected subcutaneously into the right flank with DAOY cells stably transfected with sh-circSKA3 or sh-NC. (A and B) The volume and weight of xenograft tumor were exhibited. (C and D) The levels of circSKA3 and miR-383-5p were examined by qRT-PCR. (E) The protein level of FOXMI was measured via Western blot assay. Error bar, SD; *P<0.05.

study in cervical cancer disclosed that the level of FOXMI was distinctly increased in cervical cancer, and its silencing blocked cell migration and invasion targeted by miR$214 .^{35}$ In the present research, FOXM1 was obviously enhanced in MB and verified as a candidate of miR-3835p. The expression of FOXM1 in MB was in line with the results in the past document. ${ }^{29,30}$ The promotion effect of miR-383-5p on cell apoptosis and the inhibition effect on cell proliferation, cell cycle progression, migration, and metastasis were reversed by FOXM1. Furthermore, circSKA3 silencing reduced FOXM1 expression via miR383-5p in MB. These results suggested that circSKA3 acted as a sponge of miR-383-5p, and then regulated FOXM1 expression to accelerate MB progression.

In conclusion, these data suggested that circSKA3 depletion blocked MB progression by regulating FOXM1 expression via miR-383-5p. This new regulatory pathway may shed light on the mechanism of $\mathrm{MB}$ and provide new markers for the early-stage diagnosis for MB patients.

\section{Disclosure}

The authors have no conflicts of interest to declare.

\section{References}

1. Northcott PA, Jones DT, Kool M, et al. Medulloblastomics: the end of the beginning. Nat Rev Cancer. 2012;12(12):818-834. doi:10.1038/ nrc3410
2. Pui CH, Gajjar AJ, Kane JR, Qaddoumi IA, Pappo AS. Challenging issues in pediatric oncology. Nat Rev Clin Oncol. 2011;8(9):540-549. doi:10.1038/nrclinonc.2011.95

3. Gajjar AJ, Robinson GW. Medulloblastoma-translating discoveries from the bench to the bedside. Nat Rev Clin Oncol. 2014;11 (12):714-722. doi:10.1038/nrclinonc.2014.181

4. Massimino M, Biassoni V, Gandola L, et al. Childhood medulloblastoma. Crit Rev Oncol Hematol. 2016;105:35-51. doi:10.1016/j.critrevonc.2016.05.012

5. Rutkowski S, von Hoff K, Emser A, et al. Survival and prognostic factors of early childhood medulloblastoma: an international meta-analysis. J Clin Oncol. 2010;28(33):4961-4968. doi:10.1200/ jco.2010.30.2299

6. Gajjar A, Chintagumpala M, Ashley D, et al. Risk-adapted craniospinal radiotherapy followed by high-dose chemotherapy and stem-cell rescue in children with newly diagnosed medulloblastoma (St Jude Medulloblastoma-96): long-term results from a prospective, multicentre trial. Lancet Oncol. 2006;7(10):813-820. doi:10.1016/s14702045(06)70867-1

7. Spiegler BJ, Bouffet E, Greenberg ML, Rutka JT, Mabbott DJ. Change in neurocognitive functioning after treatment with cranial radiation in childhood. $J$ Clin Oncol. 2004;22(4):706-713. doi:10.1200/jco.2004.05.186

8. Mabbott DJ, Spiegler BJ, Greenberg ML, Rutka JT, Hyder DJ, Bouffet E. Serial evaluation of academic and behavioral outcome after treatment with cranial radiation in childhood. $J$ Clin Oncol. 2005;23(10):2256-2263. doi:10.1200/jco.2005.01.158

9. Ling H, Vincent K, Pichler M, et al. Junk DNA and the long non-coding RNA twist in cancer genetics. Oncogene. 2015;34 (39):5003-5011. doi:10.1038/onc.2014.456

10. Huang T, Alvarez A, Hu B, Cheng SY. Noncoding RNAs in cancer and cancer stem cells. Chin J Cancer. 2013;32(11):582-593. doi: $10.5732 /$ cjc. 013.10170

11. De Windt LJ, Giacca M. Non-coding RNA function in stem cells and regenerative medicine. Noncoding RNA Res. 2018;3(2):39-41. doi:10.1016/j.ncrna.2018.04.004 
12. Anastasiadou E, Jacob LS, Slack FJ. Non-coding RNA networks in cancer. Nat Rev Cancer. 2018;18(1):5-18. doi:10.1038/nrc.2017.99

13. Zhang XO, Wang HB, Zhang Y, Lu X, Chen LL, Yang L. Complementary sequence-mediated exon circularization. Cell. 2014;159(1):134-147. doi:10.1016/j.cell.2014.09.001

14. Memczak S, Papavasileiou P, Peters O, Rajewsky N. Identification and characterization of circular RNAs as a new class of putative biomarkers in human blood. PLoS One. 2015;10(10):e0141214. doi:10.1371/journal.pone.0141214

15. Su H, Tao T, Yang Z, et al. Circular RNA cTFRC acts as the sponge of MicroRNA-107 to promote bladder carcinoma progression. Mol Cancer. 2019;18(1):27. doi:10.1186/s12943-019-0951-0

16. Bachmayr-Heyda A, Reiner AT, Auer K, et al. Correlation of circular RNA abundance with proliferation-exemplified with colorectal and ovarian cancer, idiopathic lung fibrosis, and normal human tissues. Sci Rep. 2015;5:8057. doi:10.1038/srep08057

17. Lv T, Miao YF, Jin K, et al. Dysregulated circular RNAs in medulloblastoma regulate proliferation and growth of tumor cells via host genes. Cancer Med. 2018;7(12):6147-6157. doi:10.1002/cam4.1613

18. Du WW, Yang W, Li X, et al. The circular RNA circSKA3 binds integrin $\beta 1$ to induce invadopodium formation enhancing breast cancer invasion. Mol Ther. 2020;28(5):1287-1298. doi:10.1016/j. ymthe.2020.03.002

19. Memczak S, Jens M, Elefsinioti A, et al. Circular RNAs are a large class of animal RNAs with regulatory potency. Nature. 2013;495 (7441):333-338. doi:10.1038/nature11928

20. Garzon R, Calin GA, Croce CM. MicroRNAs in cancer. Annu Rev Med. 2009;60:167-179. doi:10.1146/annurev.med.59.053006.104707

21. Singh SV, Dakhole AN, Deogharkar A, et al. Restoration of miR-30a expression inhibits growth, tumorigenicity of medulloblastoma cells accompanied by autophagy inhibition. Biochem Biophys Res Commun. 2017;491(4):946-952. doi:10.1016/j.bbrc.2017.07.140

22. Panwalkar P, Moiyadi A, Goel A, et al. MiR-206, a cerebellum enriched miRNA is downregulated in all medulloblastoma subgroups and its overexpression is necessary for growth inhibition of medulloblastoma cells. J Mol Neurosci. 2015;56(3):673-680. doi:10.1007/ s12031-015-0548-Z

23. Jiang J, Xie C, Liu Y, Shi Q, Chen Y. Up-regulation of miR-383-5p suppresses proliferation and enhances chemosensitivity in ovarian cancer cells by targeting TRIM27. Biomed Pharmacother. 2019;109:595-601. doi:10.1016/j.biopha.2018.10.148

24. Hu Y, Ma Y, Liu J, Cai Y, Zhang M, Fang X. LINC01128 expedites cervical cancer progression by regulating miR-383-5p/SFN axis. BMC Cancer. 2019;19(1):1157. doi:10.1186/s12885-019-6326-5
25. Xu G, Li N, Zhang Y, Zhang J, Xu R, Wu Y. MicroRNA-383-5p inhibits the progression of gastric carcinoma via targeting HDAC9 expression. Braz J Med Biol Res. 2019;52(8):e8341. doi:10.1590/ $1414-431 \times 20198341$

26. Kalinichenko VV, Major ML, Wang X, et al. Foxm1b transcription factor is essential for development of hepatocellular carcinomas and is negatively regulated by the p19ARF tumor suppressor. Genes Dev. 2004;18(7):830-850. doi:10.1101/gad.1200704

27. Yang SS, Gao Y, Wang DY, et al. Overexpression of eukaryotic initiation factor 5A2 (EIF5A2) is associated with cancer progression and poor prognosis in patients with early-stage cervical cancer. Histopathology. 2016;69(2):276-287. doi:10.1111/his.12933

28. Wang X, Jin Y, Zhang H, Huang X, Zhang Y, Zhu J. MicroRNA-599 inhibits metastasis and epithelial-mesenchymal transition via targeting EIF5A2 in gastric cancer. Biomed Pharmacother. 2018;97:473-480. doi:10.1016/j.biopha.2017.10.069

29. Priller M, Pöschl J, Abrão L, et al. Expression of FoxM1 is required for the proliferation of medulloblastoma cells and indicates worse survival of patients. Clin Cancer Res. 2011;17(21):6791-6801. doi:10.1158/1078-0432.ccr-11-1214

30. Zang W, Wang T, Wang Y, et al. Knockdown of long non-coding RNA TP73-AS1 inhibits cell proliferation and induces apoptosis in esophageal squamous cell carcinoma. Oncotarget. 2016;7 (15):19960-19974. doi:10.18632/oncotarget.6963

31. Zhou R, Wu Y, Wang W, et al. Circular RNAs (circRNAs) in cancer. Cancer Lett. 2018;425:134-142. doi:10.1016/j.canlet.2018.03.035

32. Shen PF, Chen XQ, Liao YC, et al. MicroRNA-494-3p targets CXCR4 to suppress the proliferation, invasion, and migration of prostate cancer. Prostate. 2014;74(7):756-767. doi:10.1002/ pros. 22795

33. Li XT, Wang HZ, Wu ZW, et al. miR-494-3p regulates cellular proliferation, invasion, migration, and apoptosis by PTEN/AKT signaling in human glioblastoma cells. Cell Mol Neurobiol. 2015;35 (5):679-687. doi:10.1007/s10571-015-0163-0

34. Wang X, Chen D, Gao J, et al. Centromere protein U expression promotes non-small-cell lung cancer cell proliferation through FOXM1 and predicts poor survival. Cancer Manag Res. 2018;10:6971-6984. doi:10.2147/cmar.s182852

35. Hong $\mathrm{H}$, Zhu H, Zhao S, et al. The novel circCLK3/miR-320a/ FoxM1 axis promotes cervical cancer progression. Cell Death Dis. 2019;10(12):950. doi:10.1038/s41419-019-2183-z

\section{Publish your work in this journal}

Cancer Management and Research is an international, peer-reviewed open access journal focusing on cancer research and the optimal use of preventative and integrated treatment interventions to achieve improved outcomes, enhanced survival and quality of life for the cancer patient.
The manuscript management system is completely online and includes a very quick and fair peer-review system, which is all easy to use. Visit http://www.dovepress.com/testimonials.php to read real quotes from published authors. 